\title{
MiR-144-5p, an exosomal miRNA from bone marrow-derived macrophage in type 2 diabetes, impairs bone fracture healing via targeting Smad1
}

\author{
Dong Zhang ${ }^{\dagger}$, Yifan Wu ${ }^{\dagger}$, Zonghuan Li, Hairen Chen, Siyuan Huang, Chao Jian ${ }^{*}$ and Aixi Yu ${ }^{*}$ (])
}

\begin{abstract}
Background: Patients with diabetes have an increased risk of nonunion and delayed union of fractures. Macrophages have been shown as a key player in diabetic complications. However, it remains obscure how diabetic milieu affects macrophage-derived exosomes and its implications on osteogenic differentiation of BMSCs. In this study, we aim to define the impact of diabetic milieu on macrophage-derived exosomes, role of extracellular vesicles in intercellular communication with BMSCs, and subsequent effects on osteogenic differentiation and fracture repair.
\end{abstract}

Results: The osteogenic potential and the ability of fracture repair of exosomes derived from diabetic bone marrowderived macrophages (dBMDM-exos) were revealed to be lower, as compared with non-diabetic bone marrowderived macrophages (nBMDM-exos) in vitro and in vivo. Interestingly, miR-144-5p levels were sharply elevated in dBMDM-exos and it could be transferred into BMSCs to regulate bone regeneration by targeting Smad1. In addition, the adverse effects of dBMDM-exos on the osteogenic potential and the ability of fracture repair were reversed through the suppression of miR-144-5p inhibition in vitro and vivo.

Conclusions: The results demonstrated an important role of exosomal miR-144-5p in bone regeneration, offering insight into developing new strategy for the improvement of fracture healing in patients with diabetes mellitus.

Keywords: Exosome, Macrophage, MiR-144-5p, Osteoblast, Fracture, Smad1

\section{Background}

Diabetes mellitus (DM) is a major health problem imposing serious personal and societal healthcare burdens worldwide [1]. Uncontrolled diabetes leads to multiorgan pathology, which ultimately causes disability and reduces life expectancy [2]. Over the past decades, a series of consistent studies in animals and humans have consistently demonstrated that DM adversely affects fracture-healing by disturbing the balance of bone homeostasis, and there

*Correspondence: chaojian@whu.edu.cn; yuaixi@whu.edu.cn

tDong Zhang and Yifan Wu contributed equally to this work Department of Orthopedics Trauma and Microsurgery, Zhongnan

Hospital of Wuhan University, Wuhan 430072, Hubei, China is a higher risk of delayed union and nonunion with a doubling of the time to healing of the fracture in diabetes when compared with non-diabetes [3-5].

It is increasingly recognized that impaired bone repair and regeneration in DM is regarded as the result of abnormal crosstalk between macrophage and bone mesenchymal stem cells (BMSCs) that disrupts osteogenic differentiation and fracture healing [6-10]. Macrophages diffusely distributed in the body secrete various bioactive substances, receptors and enzymes that mediate cell to cell communication, and it has been regarded as a crucial aspect of diabetic complications [8-12]. Previous studies have demonstrated that bone marrow-derived macrophages (BMDMs) original author(s) and the source, provide a link to the Creative Commons licence, and indicate if changes were made. The images or other third party material in this article are included in the article's Creative Commons licence, unless indicated otherwise in a credit line to the material. If material is not included in the article's Creative Commons licence and your intended use is not permitted by statutory regulation or exceeds the permitted use, you will need to obtain permission directly from the copyright holder. To view a copy of this licence, visit http://creativecommons.org/licenses/by/4.0/. The Creative Commons Public Domain Dedication waiver (http://creativeco mmons.org/publicdomain/zero/1.0/) applies to the data made available in this article, unless otherwise stated in a credit line to the data. 
are situated closely adjacent to BMSCs, and shape the bone microenvironment via secreting various functional biomolecules [11-13]. In addition, BMDMs have been shown to be in adverse pathological status caused via DM, and secrete osteogenesis-impeded factors including IL-1, TNF- $\alpha$, iNOS and so on, thus impairing osteogenic differentiation and increasing the probabilities of an uncontrolled regulation of fracture repair [14-19]. These factors, however, neither entirely represent the macrophages-BMSCs crosstalk under diabetic disorder nor completely account for the mechanisms underlying the impaired bone repair and regeneration under diabetic condition in DM.

Exosomes are $50-100 \mathrm{~nm}$ diameter extracellular vesicles secreted via various cell types, and derive from endosomes; they can carry abundant bioactive substances, such as noncoding RNA, mRNA, DNA, protein and other molecules [20]. Recent studies have demonstrated that they effectively mediate intercellular and interorgan communications via delivering cargos containing specific miRNAs [21]. Some miRNAs have been revealed to participate in the regulation of proliferation, migration, differentiation and apoptosis of BMSCs through modulating the expression of target genes at the post-transcriptional level, indicating that miRNAs are important regulators of bone repair and regeneration [22-24]. And there is evidence that exosomal miRNAs are capable of being stably transferred from the bone microenvironment and into BMSCs, and contribute to the process of osteogenic differentiation and fracture healing [25-28]. As such, exosomal miRNAs represent key regulators of fracture repair with significant roles at various stages of the repair process. Indeed some studies have showed that exosomal miRNAs derived from macrophages are able to influence the fracture healing via crosstalk with BMSC differentiation in vitro and in vivo [29]. Rather, the influence of BMDMs-derived exosomal miRNAs, role of DM, and their crosstalk with BMSCs remain undefined. Therefore, we aimed at evaluating the in vitro and in vivo effects of diabetic milieu on BMDMsderived exosomal miRNAs, alterations in intercellular crosstalk with BMSCs, and influence on osteogenic differentiation and fracture repair. And it may provide effective approaches and potential therapeutic targets for the treatment of impaired fracture healing in DM.

\section{Results}

\section{Evaluation of the T2DM rat model}

Our data showed that all HFD/STZ-induced rats showed 2 type diabetes mellitus. As shown in Fig. 1, there were significantly higher food intake, water consumption and urine output in T2DM group compared with the control group $(P<0.05)$. Meanwhile, rats in the T2DM group were thinner than control group $(P<0.05)$. All these are the typical symptoms of type 2 diabetes mellitus. As presented in Fig. 1, at three days after STZ injection, the random blood glucose level in T2DM group was significantly higher compared to normal group and remained higher than $16.7 \mathrm{mmol} / \mathrm{l}$ until the end of the experiment. In addition, to investigate glucose tolerance and insulin sensitivity in two groups, the IPGTT and ITT were performed. The data showed that HFD/STZ-induced rats displayed hyperglycemia in comparison with control rats during $120 \mathrm{~min}$ after glucose administration (Fig. 1). The ITT study revealed that blood glucose levels were rapidly decreased in normal group after insulin injection, whereas the blood glucose levels were reduced slowly or even not reduced in T2DM group within $30 \mathrm{~min}$. These results indicated that the successful establishment of type 2 diabetes mellitus rat models with insulin resistance and hyperglycemia.

\section{nBMDM-exos and dBMDM-exos can be internalized by BMSCs}

BMDMs were thus obtained from femurs and tibias of rats in T2DM group or normal group. The BMDMs were characterized by morphology and flow cytometry studies. Cells showed round-like shape and were positive for F4-80 and CD11b, confirming the successful isolation of BMDMs from rats (Fig. 2a, b and Additional file 1: Fig. S1). The extracted exosomes derive from nBMDMs and dBMDMs were examined by using transmission electron microscopy (TEM), dynamic light scattering (DLS) and Western blotting. TEM images showed that the particles displayed a cup- or round- shaped morphology (Fig. 2c). The particle sizes, as revealed by DLS analysis, ranged from 30 to $200 \mathrm{~nm}$ (Fig. 2d). In addition, the specific surface markers of exosomes including CD9, CD63 and TSG101 were validated by using the Western blotting analyses (Fig. 2e). These data support the successful isolation of exosomes from nBMDMs and dBMDMs. Furthermore, an exosomes uptake assay was performed, and the results demonstrated that nBMDM-exos and $\mathrm{dBMDM}$ exos could be internalized by BMSCs (Fig. 2f).

\section{dBMDM-exos hinder BMSCs osteogenesis differentiation}

We then determined the effects of nBMDM-exos and $\mathrm{dBMDM}$-exos on the BMSCs osteogenesis differentiation in vitro. After BMSCs were cocultured with $200 \mu \mathrm{g} / \mathrm{ml}$ nBMDM-exos, $200 \mu \mathrm{g} / \mathrm{ml} \mathrm{dBMDM-exos} \mathrm{or} \mathrm{equal} \mathrm{quanti-}$ ties of PBS respectively, their osteogenesis-related genes including RUNX2, ALP, collagen I, OCN were measured via Western blotting and qRT-PCR methods. As shown in Fig. 3a-c, the protein and mRNA levels of RUNX2, ALP, collagen I, OCN were downregulated in ABMDMexos groups in comparisons with other two groups. 
Furthermore, as shown by ALP staining and Alizarin red staining, the proportion of mineralization was increased by nBMDM-exos, but decreased by dBMDM-exos when compared with PBS group (Fig. 3d-f). These data indicated that dBMDM-exos may inhibit the BMSCs osteogenic differentiation.

\section{dBMDM-exos impair femoral fractur healing in a rat model}

To investigate the role of dBMDM-exos in the bone repair and regeneration in vivo, we used a rat transverse femur shaft fracture model treated with PBS, nBMDM-exos or dBMDM-exos. Western blotting and qRT-PCR analyses of samples from fracture region showed that the protein and mRNA levels of RUNX2, ALP, collagen I, OCN were significantly lower in dBMDM-exos group compared with PBS or nBMDM-exos group (Fig. 4a-c). In addition, the fracture repair at the fracture site was evaluated through X-ray and micro-CT examinations. The results indicated that compared with the PBS or nBMDM-exos group, the $\mathrm{dBMDM}$-exos group a thinner callus volume and larger fracture gap (Fig. 4d, e). Quantitative analysis of micro-CT data demonstrated that the bone volume/ total volume (BV/TV) values of the $\mathrm{ABMDM}$-exos group were significantly decreased when compared with other groups (Fig. 4f). As shown in Fig. 4g, histology images for $\mathrm{H} \& \mathrm{E}$, safranin O-fast green, Masson showed that there was a visible hindrance in the fracture healing of the femur in the rats treated with $\mathrm{ABMDM}$-exos compared with rats in other two groups. Our results demonstrated that $\mathrm{dBMDM}$-exos impaired femoral fractur healing in the rat models.

\section{The identification of differentially expressed miRNAs in nBMDM-exos and dBMDM-exos}

Becuase exosomal miRNAs play important roles in bone repair and regeneration, we further identified the differentially expressed miRNAs between nBMDM-exos and dBMDM-exos by conducting miRNA sequencing study. And as shown in Fig. 5a, b, the Volcano plot and heat map analysis displayed that a number of miRNAs were significantly upregulated in $\mathrm{ABMDM}$-exos. The top five miRNAs including miR-16-3p, miR-144-5p, miR-9a-3p, miR-1298, miR-219a-2-3p altered to the greatest degrees were validated via $\mathrm{qRT}-\mathrm{PCR}$, among which miR-144-5p was the most upregulated in dBMDM-exos (Fig. 5d). Based on the RT-qPCR results, we also found that miR144-5p was significantly enriched in dBMDMs when compared with nBMDMs, which was consistent with that in exosomes (Fig. 5e). Furthermore, these exosomes were co-cultured with BMSCs, the RT-qPCR results showed that BMSCs treated with dBMDM-exos exhibited significantly increased expression of miR-144-5p
(Fig. 5f). Together, these results suggest that miR-144-5p is elevated in ABMDM-exos and ABMDM-derived exosomal miR-144-5p can be internalized by BMSCs.

\section{miR-144-5p suppresses BMSCs osteogenesis differentiation}

To investigate the effect of miR-144-5p on BMSCs osteogenesis differentiation, BMSCs were transfected with miR-144-5p-mimic, mimic-NC, miR-144-5p-inhibitor or inhibitor-NC respectively. qRT-PCR results showed that miR-144-5p-mimic increased the levels of miR-144-5p in BMSCs, while that with miR-144-5p-inhibitor significantly decreased the levels of miR-144-5p in BMSCs (Fig. 6a). Next, the mRNA and protein levels of RUNX2, ALP, collagen I, OCN in the indicated BMSCs were determined by using qRT-PCR and Western blotting. And a significantly decreased expression in these osteogenesis related genes was observed in miR-144-5p-mimic group, but an increased expression happened in miR144-5p-inhibitor group (Fig. 6b-d). Moreover, as shown by Alizarin red and ALP staining, the mineralization was increased after miR-144-5p inhibition, while decreased following miR-144-5p overexpression (Fig. 6e-g).

\section{miR-144-5p regulates osteogenesis differentiation via targeting Smad1}

To further evaluate the mechanism by which miR-144-5p regulates the BMSCs osteogenic differentiation, four bioinformatics tools (microT, TargetScan, miRDB, and miRmap) were used to identify putative miR-144-5p target (Fig. 7a). Among these candidate target genes, Smad1 playing a key role in bone morphogenetic protein (BMP) signaling pathway was finally selected as target gene for further investigations. As shown in Fig. 7b-d, we found that overexpression of miR-144-5p significantly downregulated the expression of Smad1 at both the mRNA and protein levels, while the opposite effects were observed after the inhibition of miR-144-5p. Furthermore, we used a luciferase reporter assay to evaluate the interactions between miR-144-5p and the 3'-UTR of Smad1 in BMSCs. As shown in Fig. 7e, the luciferase activity of wt-Smad1 was decreased by miR-144-5p overexpression. However, the alterations of the luciferase activity were abolished in mut-Smad1. After confirming that miR144-5p could regulate Smad1, the miR-144-5p/ Smad1 axis on BMSCs osteogenic differentiation was further estimated. BMSCs were co-transfected with miR-144-5pinhibitor and si-Smad1, and then the osteogenic related genes and the mineralization were determined. And the results demonstrated that the mRNA and protein levels of RUNX2, ALP, collagen I, OCN and Smad1 were significantly increased via miR-144-5p inhibition, but the opposing effects on the above mRNAs and proteins were 


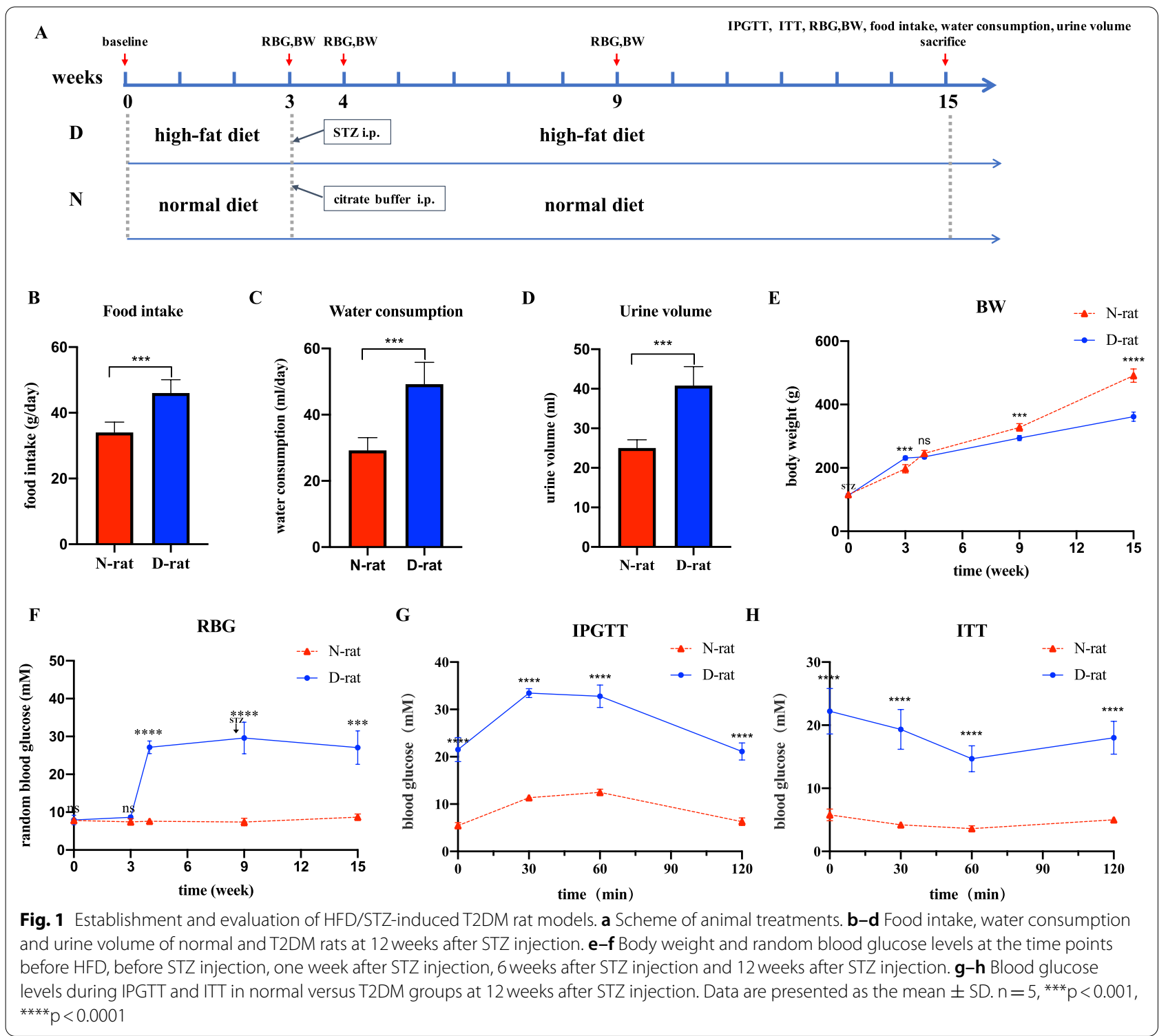

exerted via si-Smad1(Fig. $7 \mathrm{f}-\mathrm{h}$ ). And the effect of miR144-5p-inhibitor could be partly reversed by si-Smad1 (Fig. $7 \mathrm{f}-\mathrm{h}$ ). In the meanwhile, the mineralization levels were measured via Alizarin red and ALP staining, and the results showed that it was also promoted via miR144-5p-inhibitor, while inhibited by si-Smad1; the effect of miR-144-5p-inhibitor could also be partially reversed via si-Smad1(Fig. 7i-k). These results suggest that miR144-5p regulates osteogenesis differentiation via targeting Smad1.
dBMDM-derived exosomal miR-144-5p suppresses the bone repair and regeneration in vitro and vivo

After transfecting the miR-144-5p-inhibitor or inhibitor-NC into BMSCs which were co-cultured with dBMDM-exos, the expression of miR-144-5p were significantly decreased in miR-144-5p-inhibitor group, but significantly increased osteogenic related genes, Smad1 and the mineralization were detected when compared with inhibitor-NC group (Fig. 8a-f). To further define the impact of dBMDM-derived exosomal miR-144-5p 


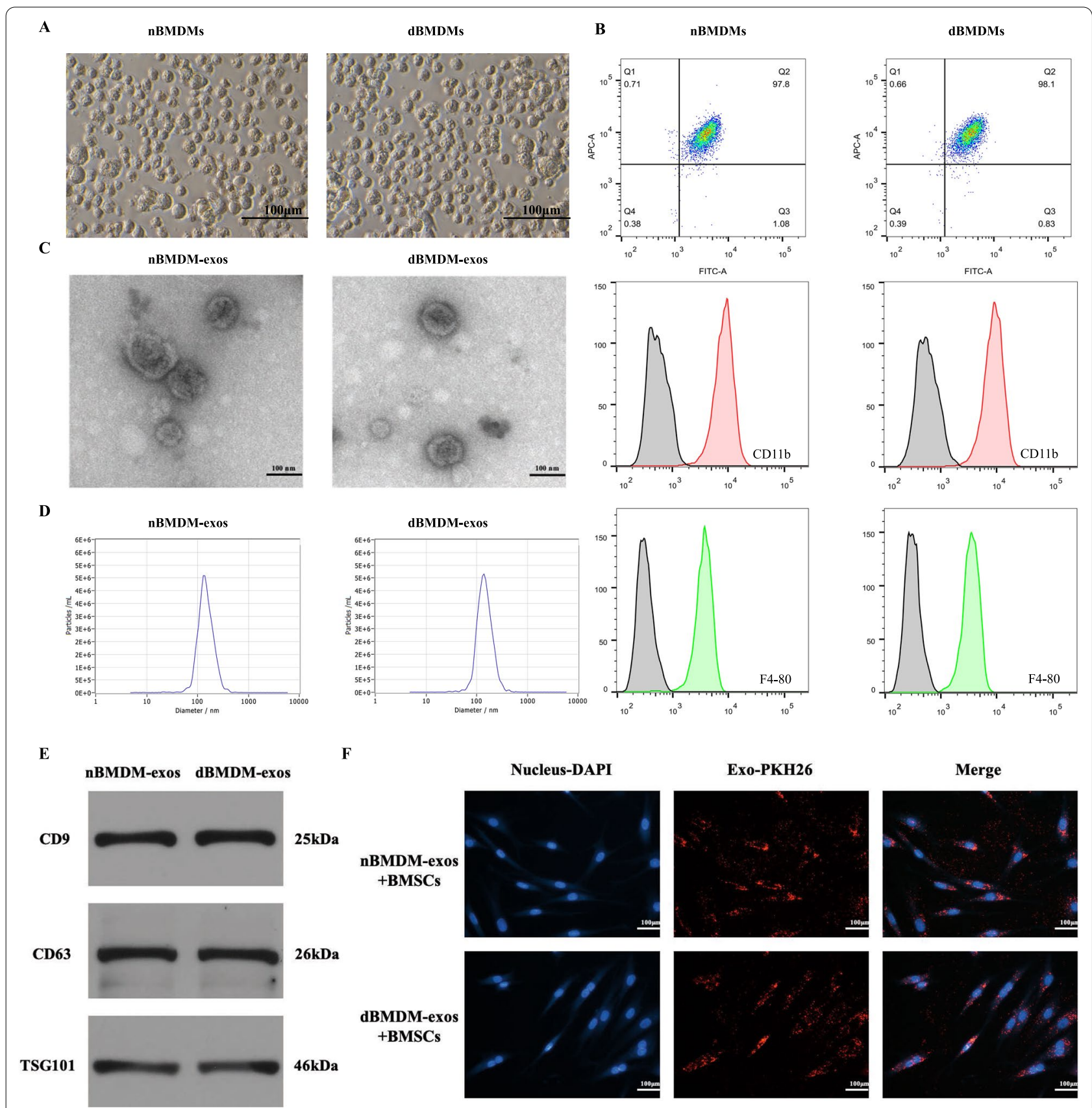

Fig. 2 Identification of BMDMs and their exosomes. a Morphology of the BMDMs under microscopy. b BMDMs surface markers F4-80 and CD11b were analyzed by flow cytometry. $\mathbf{c}$ Morphology of exosomes determined by TEM. $\mathbf{d}$ Particle size distribution of exosomes measured by DLS. e Exosome surface markers CD9, CD63 and TSG101 were examined by Western blotting. $\mathbf{f}$ BMDMs-derived exosomes were internalized by BMSCs. All experiments were repeated three times and shown are representative data

on fracture healing, a transverse femur shaft fracture model injected with $\mathrm{dBMDM}$-exos were treated with antagomir-NC or miR-144-5p-antagomir. On day 21 post-surgery, samples from fracture region were collected for qRT-PCR and Western blot analyses, and the results confirmed that miR-144-5p was downregulated, while that of Smad1 was upregulated in miR-144-5pantagomir group in comparison with antagomir-NC group (Fig. 8g-i). In addition, the protein and mRNA expression of osteogenesis related genes were also increased in miR-144-5p-antagomir group compared with antagomir-NC group (Fig. 8g-i). The X-ray images 


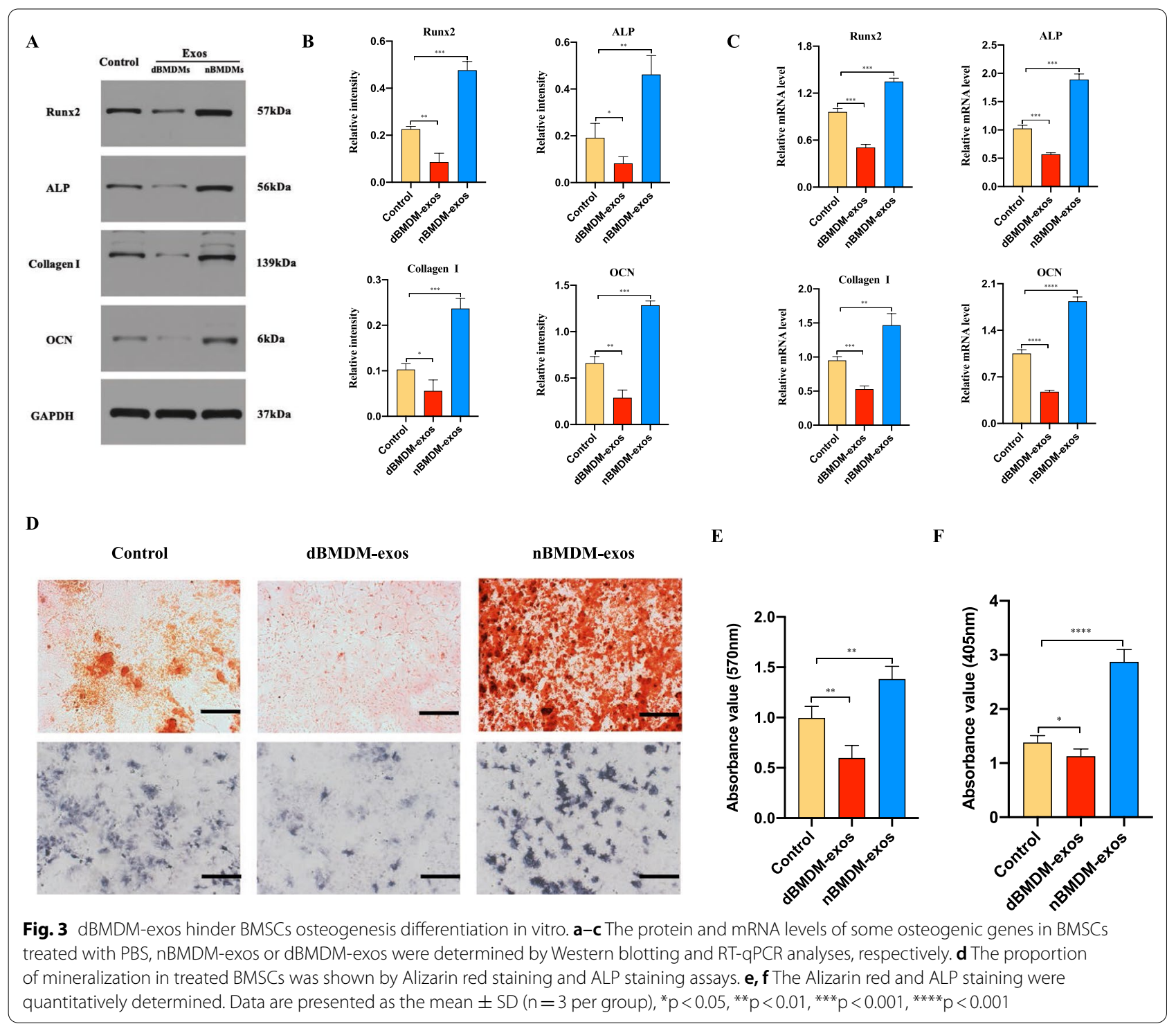

and micro-CT studies revealed a statistically decreased fracture gap and a larger callus volume happened in miR-144-5p-antagomir group, while the opposite effect happened in antagomir-NC (Fig. 8j, k). Further analysis of BV/TV offered a similar result (Fig. 8L). Moreover, histological analysis showed that there was an obvious enhancement in the fracture healing in miR-144-5pantagomir group compared with antagomir-NC group (figure $\mathrm{m}$ ). These results indicated that $\mathrm{dBMDM}$-derived exosomal miR-144-5p could suppress BMSCs osteogenesis differentiation and impair the femoral fracture healing process, and alternation of miR-144-5p could rescue the adverse effects of dBMDM-exos on bone repair and regeneration in vitro and vivo.

\section{Discussion}

DM affects hundreds of million people worldwide and impaired bone healing is an important DM-related complication which remains a threat to human health and life. Given the quickly growing incidence of diabetes especially for T2DM, it is important to improve bone healing exposed to diabetic milieu. While the mechanism of impaired fracturing healing under diabetic condition has been widely studied, no investigations focused on the macrophages-BMSCs crosstalk relied on exosomes under diabetic disorder. Recently, mounting studies have shown that exosomes can transfer miRNAs between cells, resulting in functional changes in the receiving cells and affecting bone regeneration [26, 27]. In this research, we 


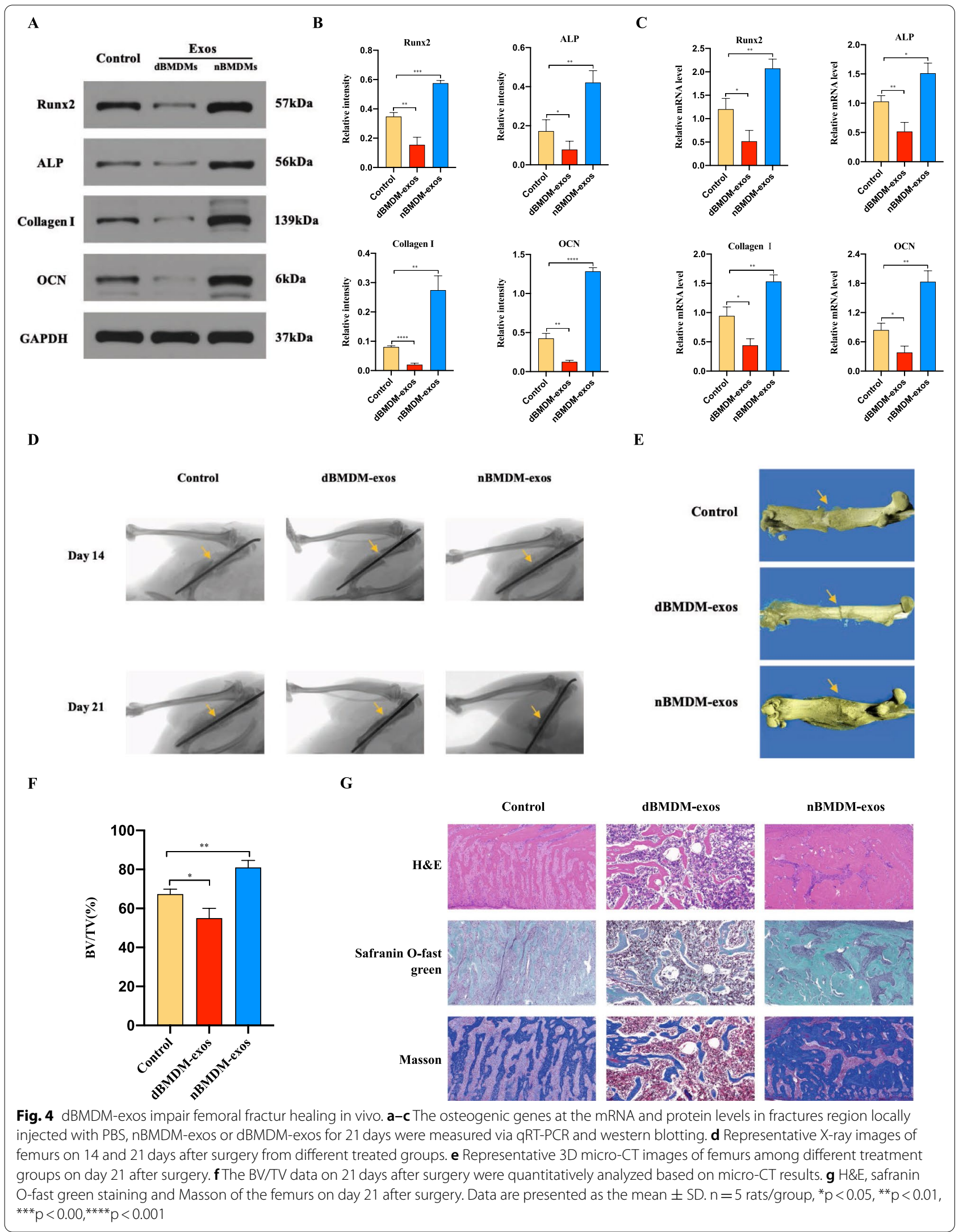




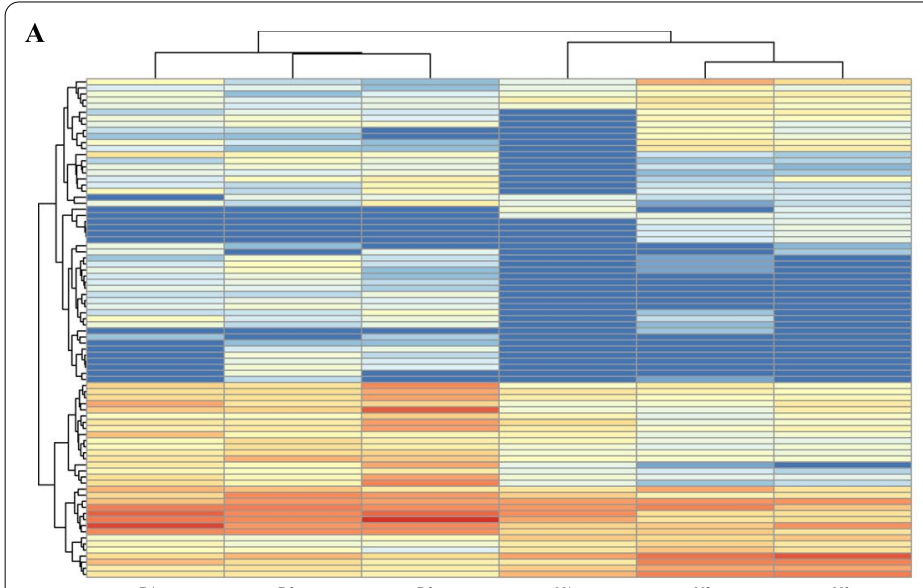

B

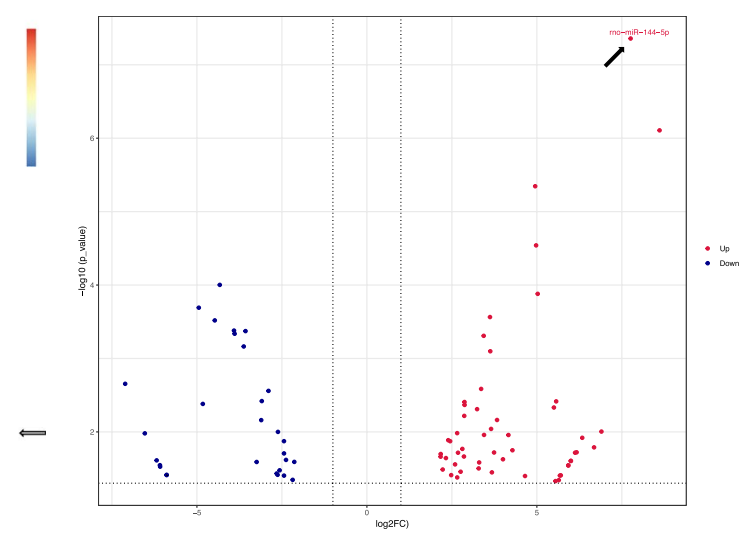

C

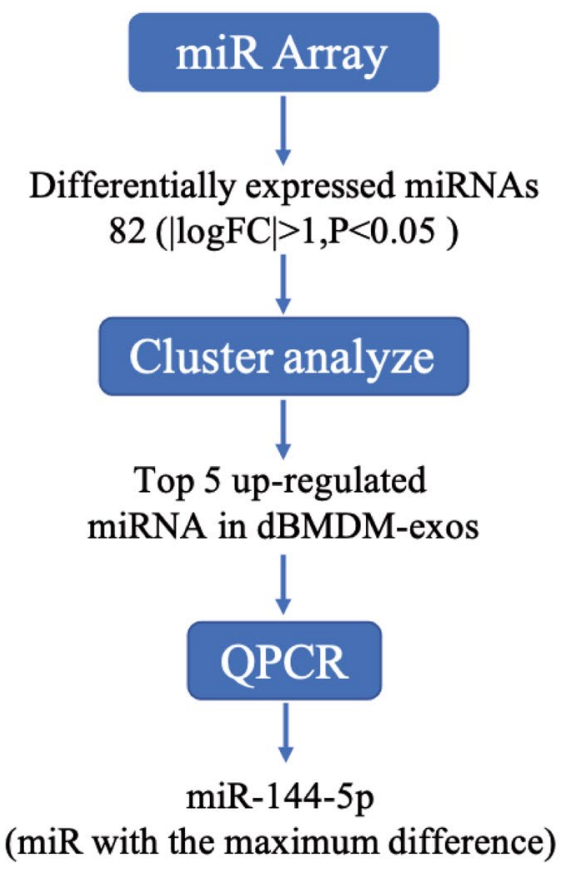

D

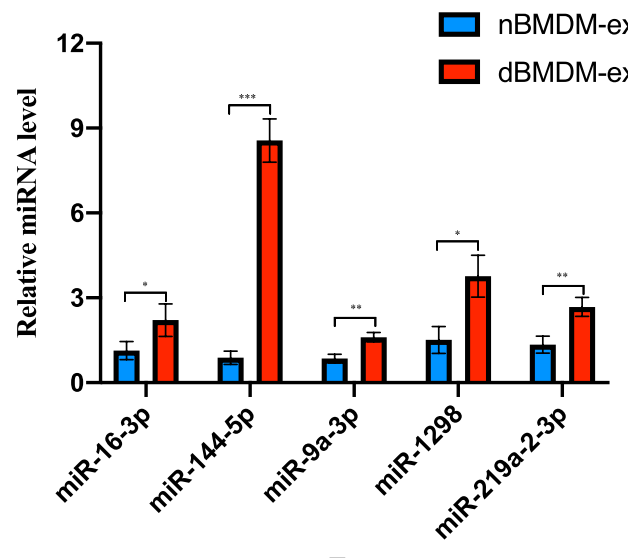

$\mathbf{E}$

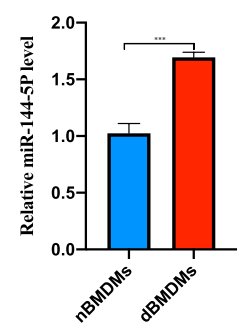

$\mathbf{F}$

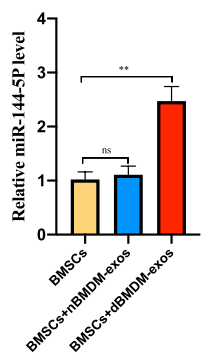

Fig. 5 MiR-144-5p was sharply increased in dBMDM-exos and it can be transferred to BMSCs via exosomes. a The heat map showed that differently expressed miRNAs $(|\log F C|>1, P<0.05$. b Volcano plot of miRNAs expressed differently in dBMDM-exos or nBMDM-exos. c The schematic diagram of microRNA screening and selection. $\mathbf{d}$ qRT-PCR analyses confirmed that, among the top 5 most upregulated miRNAs in the dBMDM-exos, miR-144-5p was increased to the greatest degree. e Comparison of miR-144-5p levels between nBMDMs and dBMDMs, as determined with qRT-PCR assay. $\mathbf{f}$ The miR-144-5p expression levels among groups following BMSCs treated with PBS, nBMDM-exos or dBMDM-exos. Data are presented as the mean \pm SD. ${ }^{*} p<0.05,{ }^{* *} p<0.01,{ }^{* * *} p<0.001$

find that the DM changes the profile of exosomal miRNAs in BMDMs, and increased exosomal miR-144-5p in $\mathrm{dBMDMs}$ can impair bone regeneration via targeting smad1 in vivo and vitro, which hold great potential to be a novel therapeutic target.

It's acknowledged that BMDMs play an essential role in modulating the osteogenesis ability of BMSCs under
DM condition, while most previous studies ascribe the results to enzymes or growth factors secreted via BMDMs. In recent years, exosomes have attracted much attention based on the ability that proteins, metabolites, and nucleic acids could be transported via exosomes into recipient cells effectively affect their biological process, and it holds potentials to identify unknown cellular 
$\mathbf{A}$

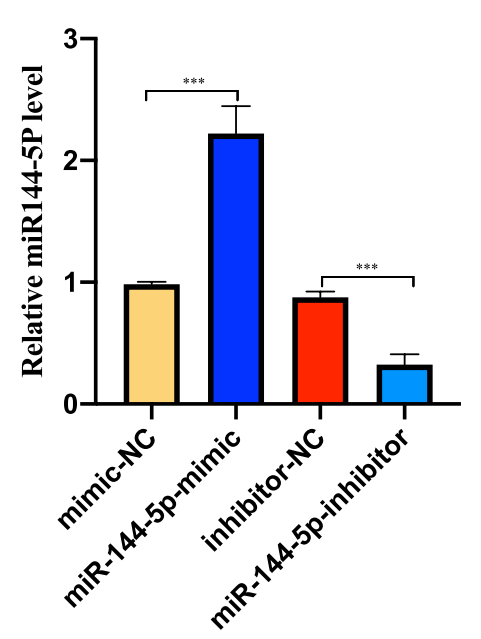

C
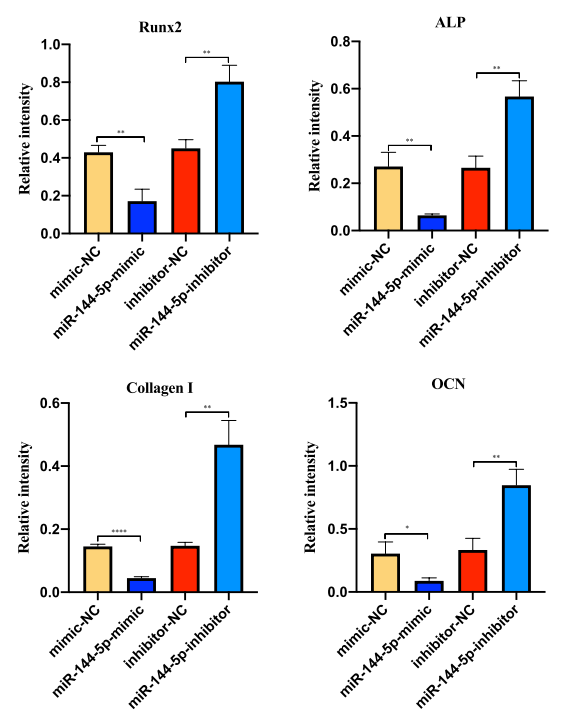

$\mathbf{E}$

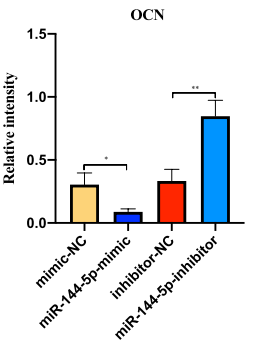

B

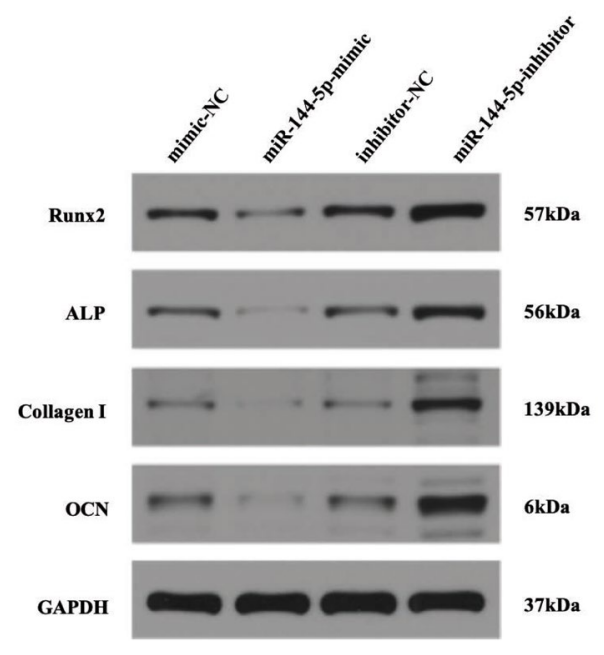

D
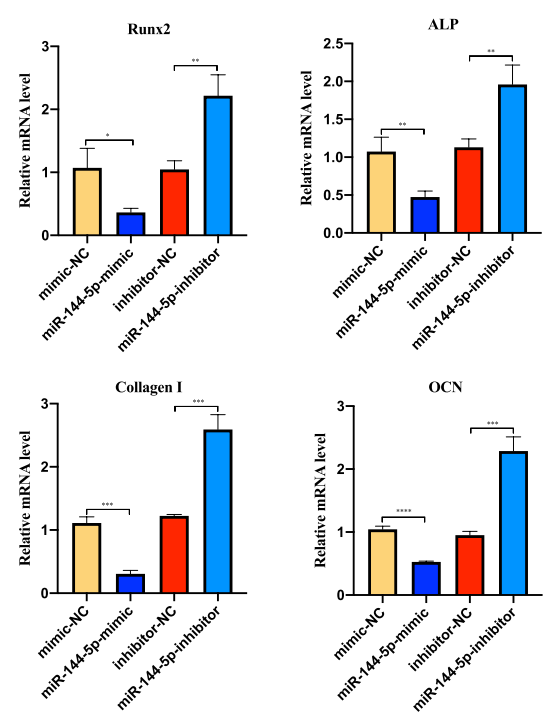

F

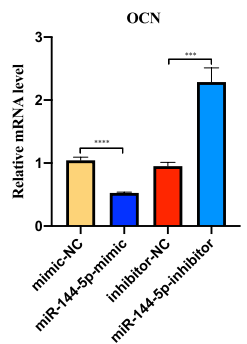

G
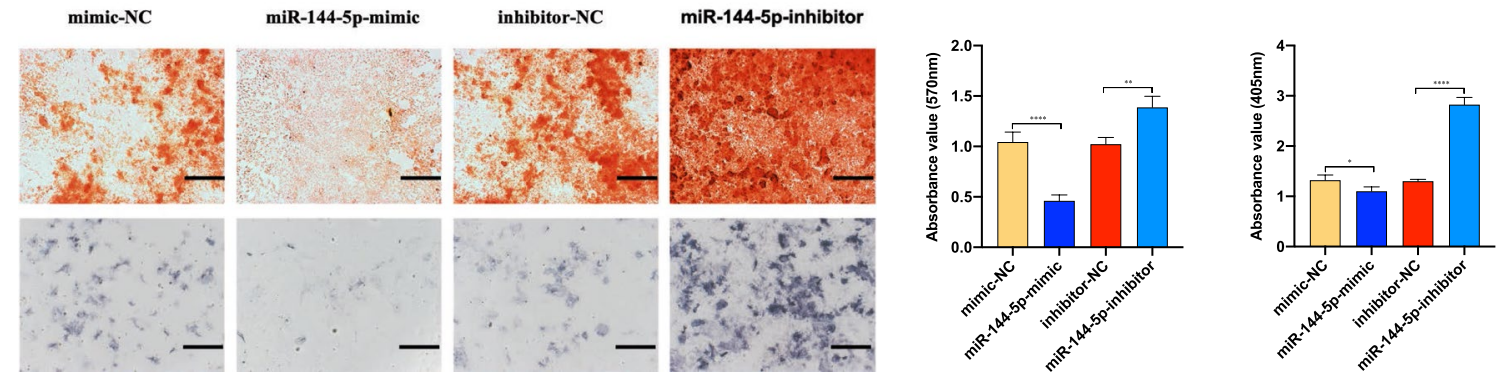

Fig. 6 Role of miR-144-5p in BMSCs osteogenesis differentiation. a Levels of miR-144-5p in BMSCs transfected with mimic-NC, miR-144-5p-mimic, inhibitor-NC or miR-144-5p-inhibitor were analyzed by qRT-PCR. b-d The osteogenic genes at the mRNA and protein levels in the transfected BMSCs were confirmed via qRT-PCR and Western blotting. $\mathbf{e}$ The proportion of mineralization in the transfected BMSCs was shown by Alizarin red staining and ALP staining assays. $\mathbf{f}, \mathbf{g}$ The statistical data of Alizarin red staining and ALP staining. Data are presented as the mean \pm SD, and all experiments were repeated three times. ${ }^{*} p<0.05,{ }^{* *} p<0.01$, ${ }^{* *} p<0.001,{ }^{* * *} p<0.001$ 
and molecular mechanisms in tissue regeneration and various disease from the perspective of cell-to-cell communication locally and between organs [21]. Furthermore, evidence have shown that macrophage-derived exosomes and their cargo might play the important role in the pathological processes of DM as well as its related complication. Wei et al. indicated that adipose tissue macrophage-derived exosomes transfer miRNAs to insulin target cells, and modulate in vivo and in vitro insulin sensitivity which can cause insulin resistance [30]. Consistent with the above findings, another study demonstrated that adipose tissue macrophage-derived exosomal miR-29a could regulate obesity-associated insulin resistance [31]. And an elegant experiment on diabetic mice showed that the pathogenesis of diabetes nephropathy was characterized by the abnormal cell-cell communication kidney-infiltrating macrophages and glomerular mesangial cells, and this cell interaction was mediated via macrophages-derived exosomes [32]. In addition, Govindappa et al. found that exosomes from BMDMs under DM condition significantly increase inflammatory and profibrogenic responses in fibroblast (in vitro) and cardiac fibrosis in mice, which reveals a novel regulatory mechanism of diabetic cardiac fibrosis and dysfunction [33]. On the basis of these findings, therefore, we assumed that exosomes derived from BMDMs exposed to DM could communicate with BMSCs via miRNAs cargo to control bone repair and regeneration in rats with DM.

To test our hypothesis, we first successfully obtained and identified BMDMs from rats with or without T2DM. Then, exosomes were isolated from the supernatant of either nBMDMs or dBMDMs, and was identified via TEM, DLS and western blotting. In accordance with the reports that exosomes can be internalized the neighboring or distant cells to modulate the function of recipient cells, our findings suggest that both of exosomes from two groups can be taken up into BMSCs, and the osteogenesis ability of BMSCs was significantly impaired after incubation with dBMDM-exos when compared with other two groups. Furthermore, our in vivo studies displayed that the process of fracture repair and regeneration in a transverse femur shaft fracture model was hindered with local injection treatment of ABMDM-exos.
Given the fact that exosomes participate in intercellular communication via containing numerous cargo molecules, and microRNAs are deeply involved in the regulation of the osteogenesis of BMSCs, we focus on the effect of exosomal miRNAs derived from BMDMs deposed to $\mathrm{DM}$ condition on the fracture healing in vitro and in vivo. Therefore, we further to identify the underlying miRNA that contribute to the negative regulation of bone repair and regeneration in $\mathrm{dBMDM}$-exos group, and miR144-5p was identified as the most upregulated miRNA in dBMDM-exos using miRNA sequencing and qRT-PCR. Furthermore, our findings also revealed that miR-144-5p was significantly upregulated in BMSCs treated with dBMDM-exos, which laid an important foundation for further researches. More importantly, our in vivo studies demonstrated that the protein levels of osteogenesis markers including RUNX2, ALP, collagen I and OCN were significantly reduced after the overexpression of miR-144-5p in BMSCs.

Four bioinformatics algorithms including microT, TargetScan, miRDB, and miRmap were used to get the potential target genes of miR-144-5p. Among all these candidate target genes, Smad1 attracts our attention. It's acknowledged that bone morphogenetic protein (BMP) is a member of the transforming growth factor-beta (TGF- $\beta$ ) superfamily which plays an essential role in the process of bone repair and regeneration; and as an immediate downstream molecules of BMP receptors, Smad1 is involved in the regulation of BMSCs osteogenesis via affecting BMP signal transduction [34-36]. And several studies have confirmed that Smad1 can be as the target gene of non-coding RNA. Based on these evidences, the relationship between miR-144-5p and Smad1 was verified using luciferase assays, and further research revealed that miR-144-5p negatively regulates the osteogenic differentiation via decreasing the expression of Smad1 in BMSCs. In addition, findings have also demonstrated that miR-144-5p-inhibitor is able to promote the osteogenic differentiation, while this effect could be partly attenuated by si-Smad1. More importantly, we further observed that the inhibition of miR-144-5p could affect the negative effect of $\mathrm{dBMDM}$-exos on bone repair and regeneration in vitro and vivo. All these results indicate

(See figure on next page.)

Fig. 7 MiR-144-5p impairs BMSCs osteogenesis differentiation via targeting Smad1. a Venn diagram of the targeting genes of miR-144-5p predicted by microT, TargetScan, miRDB, and miRmap. b-d Western blotting and qRT-PCR analysis of Smad1 protein and mRNA levels, respectively, in BMSCs transfected with mimic-NC, miR-144-5p-mimic, inhibitor-NC or miR-144-5p-inhibitor e WT and mutated miR-144-5p recognition sites within the Smad1 3' UTR were constructed for the dual-luciferase reporter assay and the results demonstrated Smad1 as a direct target gene for miR-144-5p. f-h After BMSCs were con-transfected with miR-144-5p-inhibitor or si-Smad1; the mRNAs and proteins of Smad1, RUNX2, ALP, collagen I and OCN were determined using qRT-PCR and Western blotting. I the mineralization in BMSCs following different treatments was measured via Alizarin red staining and ALP staining. j-k The statistical data of Alizarin red staining and ALP staining. Data are presented as the mean $\pm S D$, and all experiments were repeated three times. ${ }^{*} p<0.05,{ }^{* *} p<0.01,{ }^{* * *} p<0.001,{ }^{* * *} p<0.001$ 


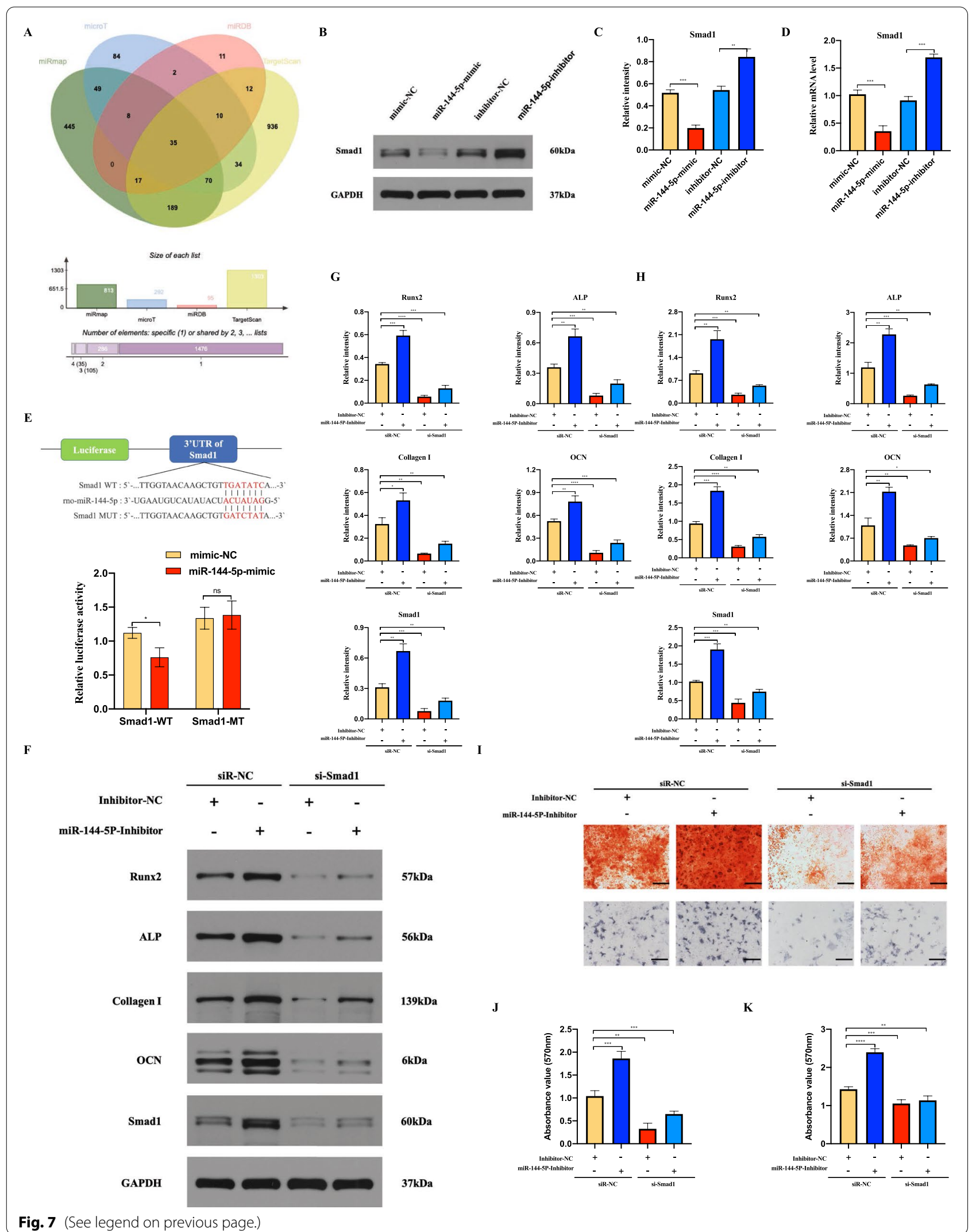


that dBMDM-derived exosomal miR-144-5p can hinder the fracture healing via decreasing the expression of Smad1.

\section{Conclusions}

Taken together, our results suggest that dBMDM-derived exosomal miR-144-5p can be transferred into BMSCs and reduce the bone repair and regeneration by suppressing the expression of Smad1 in vivo and vitro. These findings shall provide new insights into the molecular mechanism of impaired bone repair in T2DM patients and development of new remedy strategies.

\section{Methods}

\section{T2DM induction}

Ten male Sprague-Dawley rats at age 5 weeks and weighting $100-130 \mathrm{~g}$ were randomly divide into two groups: the T2DM group $(n=5)$ and the normal group $(n=5)$. The protocols of establishing T2DM rat model have been described in detail previously [37-42]. Just after 3 days of adjusting to the new environment, the rats in T2DM group were fed with a high-fat diet (HFD) containing $60 \%$ fat for 3 weeks, then they received STZ via intraperitoneal (IP) injection $(40 \mathrm{mg} / \mathrm{kg}$ in citrate buffer with $\mathrm{pH} 4.5$, Sigma, USA). The normal group fed with the normal pelleted diet for 3 weeks were intraperitoneally injected with an equal volume of citrate buffer ( $\mathrm{pH} 4.5$ ). Blood sample collected from the tail was consecutively measured for blood glucose levels. One week later, rats with more than three random blood glucose level $>16.7 \mathrm{mmol} / \mathrm{l}$ were classified as being T2DM rats. Then, to generate a long-term T2DM complication rat model, all rats in two group were returned to their cages and provided with free access to their original diets (the high-fat or control diet) and normal drinking water for 12 weeks. To evaluate this model, the food intake, water consumption, and volume of excreted urine in two groups were measured at the time point before HFD and 12 weeks after STZ injection; the body weight and random blood glucose level was recorded at the time point before HFD, before STZ injection, one week after STZ injection, 6 weeks after STZ injection and 12 weeks after STZ injection. At 12 weeks after STZ injection, IPGTT was performed. All animals were fasted overnight and given $1.5 \mathrm{~g} / \mathrm{kg}$ glucose via IP injection. Tail blood samples were taken at 0,30 , 60 , and $120 \mathrm{~min}$ for measurement of blood glucose level. In addition, ITT was carried out by administering insulin $(0.75 \mathrm{IU} / \mathrm{kg}$, ip), then blood glucose was measured at $0,30,60$, and $120 \mathrm{~min}$ after insulin injection. During all the period of the experiment, rats with blood glucose levels below $10 \mathrm{mmol} / \mathrm{l}$ were considered non-diabetic and between $10 \mathrm{mmol} / \mathrm{l}$ and $16.7 \mathrm{mmol} / \mathrm{l}$ were excluded from the study. At 12 weeks after STZ injection, animals were euthanized for the isolation of BMDMs.

\section{Cell culture}

BMDMs rats were isolated from rat models and cultured following the methods previously [43]. Briefly, bone marrow cells were flushed via PBS from the femurs and tibias in non-diabetic and diabetic groups after 12 weeks of citrate buffer or STZ injection, respectively. To obtain the BMDMs, cells were cultured in RPMI-1640 medium (without glucose) supplemented with $10 \%$ fetal bovine serum (FBS) 1\% penicillin/streptomycin, and differentiated with $25 \mathrm{ng} / \mathrm{ml}$ rat GM-CSF for 7 days, with fresh culture medium supplemented on the 4th day. And the glucose concentration of medium was adjusted using a glucose solution; to mimic the non-diabetic and diabetic conditions in vitro, cells from non-diabetic group were cultured in the medium supplemented with glucose $(5 \mathrm{mM})$, and the glucose concentration of medium used in diabetic group was $30 \mathrm{mM}$. After 7 days of culture, BMDMs were harvested for identification via flow cytometric analysis and cultured for the isolation of exosomes. BMSCs were isolated and cultured as described previously, and identified by the morphology and flow cytometric analysis [44]. The BMSCs in the passage 3-5 were used in the following experiments. The maintaining medium consists of $\alpha$-MEM supplemented with $10 \%$ FBS and $100 \mathrm{U} / \mathrm{mL}$ penicillin/streptomycin (Invitrogen). All cells were maintained in a humidified environment with $5 \% \mathrm{CO}_{2}$ at $37^{\circ} \mathrm{C}$.

\footnotetext{
(See figure on next page.)

Fig. 8 The effects of dBMDM-exos on bone repair and regeneration in vivo and vitro can be reversed through the suppression of miR-144-5p. $\mathbf{a}, \mathbf{b}$ The protein levels of Smad1 and some osteogenic genes in groups following BMSCs treated with dBMDM-exos + inhibitor-NC or dBMDM-exos + miR-144-5p-inhibitor in vitro. $\mathbf{c}$ The levels of the osteogenic genes and miR-144-5p in treated BMSCs were measured via qRT-PCR. $\mathbf{d}$. Alizarin red staining and ALP staining results in BMSCs with different treatments. e-f The statistical data of Alizarin red staining and ALP staining. g-i Levels of RUNX2, ALP, collagen I, OCN, Smad1 and miR-144-5p was determined using qRT- PCR or western blotting after dBMDM-exos + antagomir-NC or dBMDM-exos + miR-144-5p-antagomir was locally injected around the fracture site. j-k The bone formation in femur fracture was observed via X-ray images on 14, 21 days after surgery and micro-CT approach on 21 days after surgery. IThe BV/TV data on 21 days after surgery were quantitatively analyzed based on micro-CT results. $\mathbf{m} \mathrm{H} \& \mathrm{E}$, safranin O-fast green staining and Masson of the femurs on 21 days after surgery. Data are presented as the mean $\pm S D$, all cell experiments were repeated three times, and $n=5$ rats $/$ group. ${ }^{*} p<0.05$, ${ }^{* *} p<0.01$, ${ }^{* * *} p<0.001,{ }^{* * *} p<0.001$
} 


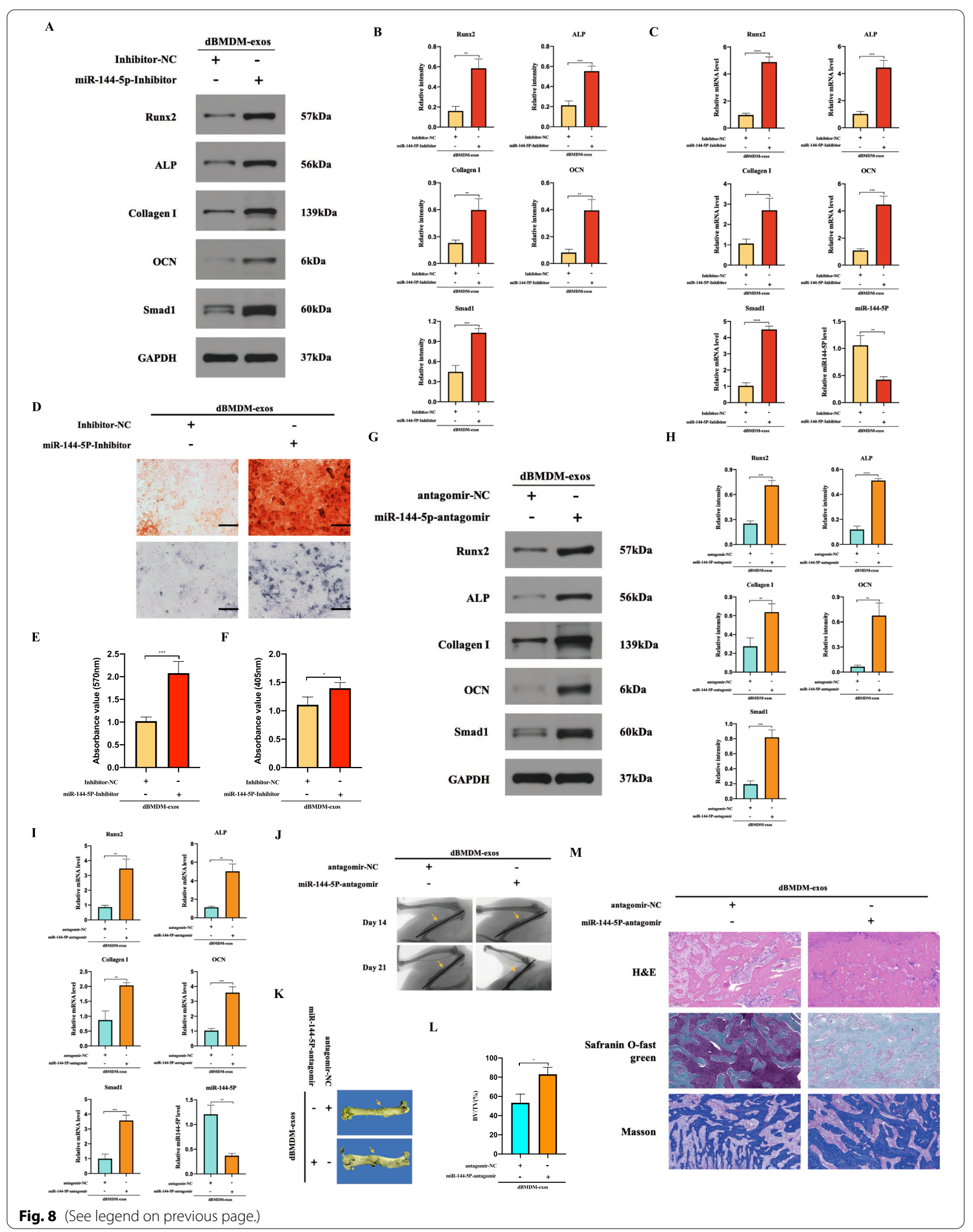


Table. 1 The sequences of primers used for PCR studies

\begin{tabular}{lll}
\hline MicroRNAs or gene name & Primer $\left(\mathbf{5}^{\prime}\right.$ to $\mathbf{3}^{\prime} \mathbf{)}$ & Primer sequence \\
\hline MiR-144-5p & ForwardReverse & GGGGGACTTACAGTATATGATGACTCAACTGGTGTCGTGGAGTC \\
Runx2 & ForwardReverse & ACTCTGCCGAGCTACGAAATGGGGACCGTCCACTGTCACTT \\
ALP & ForwardReverse & TGGACCTCATCAGCATTTGGGGGGAAGGGTCAGTCAGGTT \\
collagen I & ForwardReverse & CCGTGACCTCAAGATGTGCCGAACCTTCGCTTCCATACTCG \\
OCN & ForwardReverse & CCAGCGACTCTGAGTCTGACAAAACGGTGTGCCATAGATGC \\
Smad1U6 & ForwardReverseForwardReverse & TGTGTCACCATTCCTCGCTCCCACTCGCTTATAGTGGTAGGG \\
GAPDH & CCTGCTTCGGCAGCACATAACGCTTCACGATTTGCGT \\
\hline
\end{tabular}

\section{Flow cytometry}

BMDMs were analysed by flow cytometry. BMDMs from two groups were detected with antibodies against F4-80 (Abcam) and CD11b (Abcam). Results were analyzed by using Flowjo software.

\section{Exosome isolation and identification}

Briefly, after reaching 70-80\% confluence, BMDMs were washed three times via PBS and cultured in the media with exosome-depleted FBS for $48 \mathrm{~h}$. And the cell culture supernatant was collected and centrifuged at 100,000g for $6 \mathrm{~h}$ at $4{ }^{\circ} \mathrm{C}$. After centrifugation, samples were washed using PBS and continue undergoing ultracentrifugation at $100,000 \mathrm{~g}$ for $20 \mathrm{~min} 4{ }^{\circ} \mathrm{C}$. Then the pelleted exosomes were collected in $15 \mathrm{ml}$ of PBS and centrifuged at $4000 \mathrm{~g}$ in ultra-clear tubes to concentrate the volume to approximately $200 \mu \mathrm{l}$ for following experiments. The morphology of exosomes was observed via transmission electron microscopy (TEM; HITACHI, HT7700). And nanoparticle tracking analysis (NTA; ZetaView, Particle Metrix, Meerbusch, Germany) was used to analysis the diameter distributions of both ABMDM-exos and nBMDM-exos. In addition, specific exosome surface markers like CD9, CD63, TSG101 were identified via Western blot.

\section{Exosome uptake assay}

Briefly, BMSCs uptake of the BMDM-derived exosomes was observed through labeling the exosomes using the PKH26 Fluorescent Cell Linker Kit (Sigma) according to the manufacturer's instructions. Firstly, $20 \mu \mathrm{g}$ exosomes was mixed with $1 \mathrm{ml}$ PKH26 dye solution, incubated for $20 \mathrm{~min}$ at $25^{\circ} \mathrm{C}$, and was washed three times with PBS. Then, they were collected and resuspended via centrifuged at $110,000 \mathrm{~g}$ for $20 \mathrm{~min}$ at $4{ }^{\circ} \mathrm{C}$. Finally, PKH26labeled exosomes were added to DAPI-labeled BMSCs and cultured for $12 \mathrm{~h}$ before being observed via a confocal fluorescence microscope.

\section{MiRNA sequencing}

Samples were processed according to methods described previously [45]. Total RNA was isolated from exosomes using TRIzol reagent (Invitrogen, CA, USA) according to the manufacturer's protocol. The quality and quality of RNA samples were assessed with a NanoDrop ND-1000 and Agilent 2100 Bioanalyzer, respectively. Total RNA from each sample was used to prepare miRNA library using NEBNext ${ }^{\circledR}$ Multiplex Small RNA Library Prep Set for Illumina ${ }^{\circledR}$ (NEB) according to the Illumina small RNA sample preparation protocol. In short, adapters were ligated to the $3^{\prime}$ and $5^{\prime}$ ends of all miRNAs, and cDNA was synthesized by reverse transcription, then the cDNA samples were amplified by PCR Reaction. After library amplification, a cleanup of the miRNA library was performed to choose the fragment ranged from 135 to $155 \mathrm{bp}$ (corresponding to 15-35 nt small RNAs). Next, the quality and quantity of the library was carefully verified in automated electrophoresis, performed on Agilent 2100 Bioanalyzer. And the libraries were denatured as single-stranded DNA molecules, captured on Illumina flow cells, amplified in situ as clusters and finally sequenced for 50 cycles on an Illumina NextSeq 500 (Illumina, CA, USA) system according to the manufacturer's instructions. Differentially expressed miRNA were then identified at a criteria of fold change $>1.0$ or $<1.0$ and a $p$ value $<0.05$, and demonstrated via the heat map and the volcano plot.

\section{BMSCs osteogenic differentiation}

The BMSCs were seeded into 6-well plates and was used for osteogenic differentiation induction via a specific osteogenic induction medium (OIM, OriCell, Cyagen Biosciences, Guangzhou, China). The entire inducing process lasted 14days. For evaluating the effect of exosomes on osteogenic differentiation, $200 \mu \mathrm{l}$ exosomes $(200 \mu \mathrm{g} / \mathrm{mL})$ and equal volume PBS were added to osteogenic induction medium and refreshed every three days. In addition, BMSCs were transfected with siSMAD1, miR-144-5p mimic, miR-144-5p inhibitor and NC via Lipofectamine 3000 (Invitrogen) when 
cells confluency reached $70-80 \%$. The reagents of siRNA, miR-144-5p-mimic, miR-144-5p-inhibitor and miR-144-5p-NC were synthesized by RiboBio (Guangzhou, China), and the sequences are listed in Table 1. To evaluate the level of osteogenic differentiation, alkaline phosphatase (ALP) staining, alizarin red staining, quantitative reverse transcription polymerase chain reaction (qRT-PCR) and western blot were performed on day 14 .

\section{ALP activity and mineralization assessment}

To identify the osteogenic differentiation, BMSCs were induced for 14days with different treatment, and measured via ALP staining and alizarin red staining. According to the protocol, BMSCs were washed three times with PBS, fixed with $4 \%$ paraformaldehyde for $20 \mathrm{~min}$, and then stained with ALP staining or alizarin red staining for $30 \mathrm{~min}$ at $25^{\circ} \mathrm{C}$. After staining, the BMSCs were washed with PBS and observed using microscopy. Absorbance was then measured at $570 \mathrm{~nm}$ or $405 \mathrm{~nm}$.

\section{Luciferase reporter assay}

After BMSCs were added to 24-well plates at a density of $0.5-2 \times 10^{5}$ each well for $24 \mathrm{~h}$, dual-luciferase vectors (pGL6-miR-Smad1-WT-3' UTR, pGL6-miR-Smad1Mut-3'UTR) were transfected into them together with miR-144-5p mimics or mimic-NC. And the luciferase activity was examined using Dual-Luciferase Reporter Assay System (Promega) after $48 \mathrm{~h}$ post-transfection. Ultimately, the results were normalized to Renilla luciferase activity.

\section{The transverse femur shaft fracture establishment and treatment}

Rat transverse femur shaft fracture model was created as follows. All rats were conducted under general anesthesia with intraperitoneal injection of $60 \mathrm{mg} / \mathrm{kg}$ ketamine hydrochloride before surgical procedures. After the lower limb of the rat was shaved and disinfected, a 3-cm incision was created under sterile conditions. To expose the femurs, the soft tissues were pushed aside while the overlying periosteum was carefully preserving. And a transverse femur shaft fracture was conducted using an oscillating saw before a Kirschner's wire with 1-mm in diameter was inserted into the femur for intramedullary fixation. Finally, the subcutaneous tissue and the skin were closed using 5-0 nylon suture. After fracture, $200 \mu \mathrm{L}$ PBS and equivalent volumes of nBMDMexos, dBMDM-exos, dBMDM-exos + NC-antagomir, dBMDM-exos + miR-144-5p-antagomir were respectively injected around the fracture site at the time point $1,3,5,7$ day after surgery. And X-ray images of fracture femurs were taken at day 14 and 21 after surgery. In addition, at 21 days after operation, samples were harvested for histological analysis, micro computed tomography (micro-CT), qRT-PCR and western blot analysis.

\section{Radiographic analysis}

A small animal in vivo imaging system (Bruker Xtreme $\mathrm{BI}$ ) was used to obtain X-ray images to observe fracture regions. After rats were subjected to internal fixation removal at 21 days after surgery, all femurs were fixed with $4 \%$ paraformaldehyde for $24 \mathrm{~h}$ at $4{ }^{\circ} \mathrm{C}$. Then samples were scanned via performing a micro-CT system (SkyScan1276, Bruker, Belgium) at a resolution of $40 \mu \mathrm{m}$ with $63 \mathrm{kV}$ and $200 \mu \mathrm{A}$. After scanning, we constructed 3D structures of femurs and calculated the new bone volume/total volume (BV/TV) to assess bone regeneration in the fracture site.

\section{qRT-PCR analysis}

Total RNA was extracted from exosomes, BMSCs induced for 14days or callus tissues harvested from fracture regions at 21 days after surgery using TRIpure Extraction Reagent (EP013, ELK Biotechnology). The cDNA was reverse transcribed using EntiLink $^{\text {TM }}$ 1st Strand cDNA Synthesis Kit (Eq. 003, ELK Biotechnology) following the manufacturer's instructions. And the qRTPCR for mRNAs and miRNAs was performed on a StepOne $^{\mathrm{TM}}$ Real-Time PCR System (Life technologies) using EnTurbo $^{\mathrm{TM}}$ SYBR Green PCR SuperMix (EQ001, ELK Biotechnology). The relative expression levels of mRNA or miRNA were normalized to those of GAPDH or U6 and evaluated using the $2^{-\Delta \Delta} \mathrm{CT}$ method. The primers used for qRT-PCR are listed in Table 1.

\section{Western blot analysis}

Total protein was isolated from exosomes, BMSCs induced for 14 days or callus tissues harvested from fracture regions at 21 days after surgery using RIPA (Aspen) in accordance with the manufacturer's protocol. After the concentrations of protein samples were detected using BCA (Aspen), equal amounts of protein samples were separated by sodium dodecyl sulfate-polyacrylamide gel electrophoresis (the $15 \%$ concentration for OCN; the $12 \%$ concentration for $\mathrm{CD} 9, \mathrm{CD} 63$; the $10 \%$ concentration for TSG101, RUNX2, ALP and Smad1; the $8 \%$ concentration for collagen I), transferred to a polyvinylidene fluoride (PVDF) membrane, and incubated with $5 \%$ bovine serum albumin for $1 \mathrm{~h}$ at $25^{\circ} \mathrm{C}$. And PageRuler ${ }^{\mathrm{TM}}$ Prestained Protein Ladder (ThermoFishe, 26,617) was used as a molecular weight marker. Next, the membranes were incubated overnight at $4{ }^{\circ} \mathrm{C}$ with primary antibodies specific for CD9 (1:1000, Abcam, ab92726), CD63 (1:500, Santa, sc-5275), TSG101 (1:1000, Abcam, ab125011), 
RUNX2 (1:1000, Abcam, ab236639), ALP (1:1000, Bioss, bsm-52252R), collagen I (1:500, Abcam, ab260043), OCN (1:500, Santa, sc-390,877), Smad1 (Abcam100, Abcam, ab53745) and GAPDH (1:10,000, Abcam, ab37168). Then, they were stained with appropriate secondary antibodies at $1: 2,000$ for $30 \mathrm{~min}$. The visualization of the reacting bands was performed via ECL reagent (Thermo Fisher Scientific). And the protein bands were quantified using AlphaEaseFC (Alpha Innotech, San Leandro, CA) software. The original WB image of the osteogenic related proteins was shown in Additional file 2: Fig. S2.

\section{Histological analysis}

The femurs of rats from different groups were collected 21 days after surgery. Samples were fixed with buffered paraformaldehyde for $48 \mathrm{~h}$, and then decalcified with $20 \%$ EDTA at $25^{\circ} \mathrm{C}$ for 25 days. The samples were embedded in paraffin, sectioned along the longitudinal axis, and stained with H\&E, safranin O-fast green, Masson for histological analysis. Sections were then imaged with a microscope.

\section{Statistical analysis}

Values were presented as mean $\pm \mathrm{SD}$, and analyzed with GraphPad Prism 8.0 (Graph- Pad Software, CA, USA). All experiments were repeated at least three times. Student's t-test was used to analyze the two independent groups. A value of $\mathrm{P}<0.05$ was considered as statistically significant.

\section{Abbreviations}

D: T2DM group; N: Normal group; RBG: Random blood glucose; BW: Body weight; IPGTT: Intraperitoneal glucose tolerance test; ITT: Insulin tolerance test; i.p.: Intraperitoneal; STZ: Streptozocin; BMDM: Bone marrow-derived macrophages; nBMDMs: Bone marrow-derived macrophages from rats under normal condition; dBMDMs: Bone marrow-derived macrophages from rats under diabetic condition; Exos: Exosomes; dBMDM-exos: Exosomes derived from dBMDMs; nBMDM-exos: Exosomes derived from nBMDMs; BMSCs: Bone mesenchymal stem cells; TEM: Transmission electron microscopy; DLS: Dynamic light scattering; Runx2: Recombinant runt related transcription factor 2; ALP: Alkaline phosphatase; OCN: Osteocalcin.

\section{Supplementary Information}

The online version contains supplementary material available at https://doi. org/10.1186/s12951-021-00964-8.

Additional file 1: Fig. S1. BMDMs were identified via flow cytometry.

Additional file 2: Fig. S2. The original WB image of the osteogenic related proteins.

\section{Authors' contributions}

$A Y, C J$, and DZ designed research; DZ and YW analyzed data; DZ, YW, HC, and SH performed research; DZ and YW wrote the paper. All authors read and approved the final manuscript.

\section{Funding}

This work was supported by the Trauma Microsurgery Clinical Medical Research Center of Hubei Province and the Health Commission of Hubei Province Medical Leading Talent Project (Grant No. LJ20200405).

\section{Availability of data and materials}

All data generated or analyzed during this study are included in this published article.

\section{Declarations}

\section{Ethics approval and consent to participate}

Rats in this study were purchased from the laboratory Animal Center of Wuhan University (China). All animal experiments were performed under the guidance of the Institutional Animal Care and Use Committee (IACUC) of Wuhan University. The experimental protocol was approved by the Committee on the Ethics of Animal Experiments of Wuhan University, Wuhan, China (Approval number:WP2020-08023). All efforts were made to minimize animal suffering.

\section{Consent for publication}

All authors agree to be published.

\section{Competing interests}

All authors declared no conflict of interests.

Received: 18 May 2021 Accepted: 13 July 2021

Published online: 30 July 2021

\section{References}

1. Roglic G. WHO Global report on diabetes: a summary. Int J Noncommun Dis. 2016;1(1):3-8.

2. Association AD. Diagnosis and classification of diabetes mellitus. Diabetes Care. 2010;33(Suppl 1):S62.

3. Henderson $\mathrm{S}$, et al. Bone quality and fracture-healing in type-1 and type-2 diabetes mellitus. J Bone Joint Surg. 2019;101(15):1399-410.

4. Gaston MS, Simpson AHRW. Inhibition of fracture healing. J Bone Joint Surg Br Vol. 2007;89(12):1553-60.

5. Ogasawara A, et al. Molecular basis for affected cartilage formation and bone union in fracture healing of the streptozotocin-induced diabetic rat. Bone. 2008;43(5):832-9.

6. Shimoide T, et al. Role of macrophages and plasminogen activator inhibitor- 1 in delayed bone repair in diabetic female mice. Endocrinology. 2018;159(4):1875-85.

7. Hu Z, et al. Immunomodulatory ECM-like Microspheres for accelerated bone regeneration in diabetes mellitus. ACS Appl Mater Interfaces. 2018;10(3):2377-90.

8. Albiero $\mathrm{M}$, et al. Bone marrow macrophages contribute to diabetic stem cell mobilopathy by producing oncostatin M. Diabetes. 2015;64(8):2957-68.

9. Tesch $\mathrm{GH}$. Role of macrophages in complications of type 2 diabetes. Clin Exp Pharmacol Physiol. 2007;34(10):1016-9.

10. Knipper JA, Ding $X$, Eming SA. Diabetes impedes the epigenetic switch of macrophages into repair mode. Immunity. 2019;51(2):199-201.

11. Li Y, et al. Bone marrow macrophage M2 polarization and adiposederived stem cells osteogenic differentiation synergistically promote rehabilitation of bone damage. J Cell Biochem. 2019;120(12):19891-901.

12. Ayala TS, et al. High glucose environments interfere with bone marrowderived macrophage inflammatory mediator release, the TLR4 pathway and glucose metabolism. Sci Rep. 2019;9(1):11447.

13. Alexander KA, et al. Osteal macrophages promote in vivo intramembranous bone healing in a mouse tibial injury model. J Bone Miner Res. 2011;26(7):1517-32

14. Pajarinen J, et al. Mesenchymal stem cell-macrophage crosstalk and bone healing. Biomaterials. 2018;196:80-9. 
15. Tessaro FHG, et al. Macrophages from a type 1 diabetes mouse model present dysregulated PI3K/AKT, ERK $1 / 2$ and SAPK/JNK levels. Immunobiology. 2019;225(2):151879.

16. Murray CE, Coleman CM. Impact of diabetes mellitus on bone health. Int J Mol Sci. 2019;20(19):4873.

17. Sinder BP, Pettit AR, Mccauley LK. Macrophages: their emerging roles in bone. J Bone Min Res. 2015;30(12):2140-9.

18. Ma H, Liu G, Ding W, Wu Y, Cai L, Zhao Y. Diabetes-induced alteration of F4/80+ macrophages: a study in mice with streptozotocin-induced diabetes for a long term. J Mol Med. 2008;86(4):391-400.

19. Muñoz J, et al. Macrophage polarization and osteoporosis: a review. Nutrients. 2020;12:10.

20. Pegtel DM, Gould SJ. Exosomes. Annu Rev Biochem. 2019;88:487-514.

21. Kalluri R, LeBleu VS. The biology, function, and biomedical applications of exosomes. Science. 2020;367:6478.

22. Gennari L, Bianciardi S, Merlotti D. MicroRNAs in bone diseases. Osteoporos Int. 2017;28(4):1191-213.

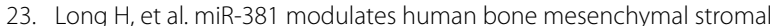
cells (BMSCs) osteogenesis via suppressing Wnt signaling pathway during atrophic nonunion development. Cell Death Dis. 2019:10(7):470.

24. Takahara $\mathrm{S}$, et al. Altered expression of microRNA during fracture healing in diabetic rats. Bone Joint Res. 2018;7(2):139-47.

25. Qin Y, et al. Bone marrow stromal/stem cell-derived extracellular vesicles regulate osteoblast activity and differentiation in vitro and promote bone regeneration in vivo. Sci Rep. 2016:6:21961.

26. XuT, et al. Exosomal miRNA-128-3p from mesenchymal stem cells of aged rats regulates osteogenesis and bone fracture healing by targeting Smad5. J Nanobiotechnology. 2020;18(1):47.

27. Zhu Y, et al. Impaired bone regenerative effect of exosomes derived from bone marrow mesenchymal stem cells in type 1 diabetes. Stem Cells Transl Med. 2019:8(6):593-605.

28. Zhu S, et al. Coupling factors and exosomal packaging microRNAs involved in the regulation of bone remodelling. Biol Rev Camb Philos Soc. 2018;93(1):469-80.

29. Xiong Y, et al. M2 Macrophagy-derived exosomal miRNA-5106 induces bone mesenchymal stem cells towards osteoblastic fate by targeting salt-inducible kinase 2 and 3. J Nanobiotechnology. 2020;18(1):66.

30. Ying W, et al. Adipose tissue macrophage-derived exosomal miRNAs can modulate in vivo and in vitro insulin sensitivity. Cell. 2017; 171(2): 372-384.

31. Liu T, et al. Adipose tissue macrophage-derived exosomal miR-29a regulates obesity-associated insulin resistance. Biochem Biophys Res Commun. 2019;515(2):352-8.
32. Zhu QJ, et al. Exosomes from high glucose-treated macrophages activate glomerular mesangial cells via TGF- $\beta 1 /$ Smad3 pathway in vivo and in vitro. Faseb J. 2019;33(8):9279-90.

33. Govindappa PK, et al. Targeting exosome-associated human antigen $R$ attenuates fibrosis and inflammation in diabetic heart. Faseb J. 2020;34(2):2238-51.

34. Cao X, Chen D. The BMP signaling and in vivo bone formation. Gene. 2005;357(1):1-8.

35. Wang $M$, et al. Smad1 plays an essential role in bone development and postnatal bone formation. Osteoarthr Cartil. 2011;19(6):751-62.

36. Nickel J, Mueller TD. Specification of BMP Signaling. Cells. 2019;8:12.

37. Sohrabipour $\mathrm{S}$, et al. GABA dramatically improves glucose tolerance in streptozotocin-induced diabetic rats fed with high-fat diet. Eur J Pharmacol. 2018;826:75-84.

38. Furman BL. Streptozotocin-induced diabetic models in mice and rats. Curr Protoc Pharmacol. 2015;70:5.47.1-5.47.20.

39. Yu S, et al. Treatment with adipose tissue-derived mesenchymal stem cells exerts anti-diabetic effects, improves long-term complications, and attenuates inflammation in type 2 diabetic rats. Stem Cell Res Ther. 2019:10(1):333.

40. Kowluru RA. Retinopathy in a diet-induced type 2 diabetic rat model and role of epigenetic modifications. Diabetes. 2020;69(4):689-98.

41. Zhang $M$, et al. The characterization of high-fat diet and multiple lowdose streptozotocin induced type 2 diabetes rat model. Exp Diabetes Res. 2008;2008:704045.

42. Reed MJ, et al. A new rat model of type 2 diabetes: the fat-fed, streptozotocin-treated rat. Metabolism. 2000:49(11):1390-4.

43. Toda G, et al. Preparation and culture of bone marrow-derived macrophages from mice for functional analysis. STAR Protoc. 2021;2(1):100246

44. Maridas DE, et al. Isolation, culture, and differentiation of bone marrow stromal cells and osteoclast progenitors from mice. J Vis Exp. 2018;131:1.

45. Feng $Y$, et al. Exosome-derived miRNAs as predictive biomarkers for diffuse large B-cell lymphoma chemotherapy resistance. Epigenomics. 2019;11(1):35-51.

\section{Publisher's Note}

Springer Nature remains neutral with regard to jurisdictional claims in published maps and institutional affiliations.
Ready to submit your research? Choose BMC and benefit from:

- fast, convenient online submission

- thorough peer review by experienced researchers in your field

- rapid publication on acceptance

- support for research data, including large and complex data types

- gold Open Access which fosters wider collaboration and increased citations

- maximum visibility for your research: over 100M website views per year

At BMC, research is always in progress.

Learn more biomedcentral.com/submissions 